Conclusions: ROC analyses demonstrated predictive validity identifying cognitive impairment and dementia in Māori and non-Māori.

\section{INSTRUMENTED TRAIL-MAKING TASK (ITMT) TO ASSESS DUAL-TASK AND COGNITIVE IMPAIRMENT IN ELDERLY}

H. Zhou ${ }^{1}$, M. Sabbagh 2 , R. Wyman ${ }^{3}$, C. Liebsack ${ }^{3}$, M. Kunik ${ }^{1}$, B. Najafi ${ }^{1}$, 1. Baylor College of Medicine, Houston, Texas, 2. Barrow Neurological Institute, Phoenix, Arizona, 3. Banner Sun Health Research Institute, Sun City, Arizona

Current modalities to examine cognitive impairment are often unsuitable for busy clinics, subjective, or insensitive to evaluate dual-task cost, which is essential to isolate the cognitive control component of locomotion. In this study we an innovative instrumented trail making task (iTMT) based on wearable sensor and human-machine interface to evaluate motor-cognitive performance in older adults.

Thirty healthy age-matched control, Mild Cognitive Impairment (MCI), and Alzheimer's Disease ( $\mathrm{AD}$ ) subjects were recruited. Participants were asked to perform iTMT tests with different cognitive-challenge levels: $\mathrm{iTMT}_{\text {fixed }}$, $\mathrm{iTMT}_{\text {random }}$, and $\mathrm{iTMT}$ number-leter . Conventional TMT A\&B, Montreal Cognitive Assessment (MoCA), and dual-task cost (DTC) were used as references.

Between groups difference was more pronounced, when using iTMT $_{\text {numberleterer, }}$, with average completion time of $26.3 \pm 12.4 \mathrm{~s}, 37.8 \pm 14.1 \mathrm{~s}$, and $61.8 \pm 34.1 \mathrm{~s}$, respectively, for healthy, MCI, and AD groups $(p=0.006)$. Pairwise comparison suggested strong effect sizes to separate between $\mathrm{AD}$ and healthy $(d=1.384, p=0.001)$ and between MCI and AD $(d=0.920, p=0.029)$. Significant correlation was observed when comparing iTMT iTumberletrer $_{\text {with }}$ MoCA $(r=-0.598$, $p=0.001)$, TMT A $(r=0.519 p=0.006)$, TMT B $(r=0.666$, $p<0.001)$, and DTC $(r=0.713, p<0.001)$. Interestingly, no noticeable correlation was observed between DTC with MoCA or TMT.

This study demonstrated proof of concept of a simple, safe, and practical iTMT system with promising results to identify cognitive and dual-task ability impairment among older adults including those suffering from $\mathrm{MCI}$ and $\mathrm{AD}$. Future studies are warranted to confirm these observations in larger samples and iTMT's ability to track cognitive decline over time.

\section{EXECUTIVE FUNCTION AND LIFE-SPACE MOBILITY IN OLD AGE}

T. Poranen-Clark ${ }^{2}$, M. von Bonsdorff ${ }^{2,3}$, M. Rantakokko ${ }^{2}$, E. Portegijs ${ }^{2}$, J. Eronen ${ }^{2}$, J.G. Eriksson ${ }^{3,1,4}$, T. Rantanen ${ }^{2}$, A. Viljanen ${ }^{2}, 1$. University of Helsinki, Helsinki, Finland, 2. University of Jyväskylä, Jyväskylä, Finland, 3. Folkhälsan, Helsinki, Finland, 4. National Institute of Health and Welfare, Helsinki, Finland

Mobility is important for maintaining independence and active participation in old age. Life-space mobility assessment incorporates the extent of mobility in terms of the distance from home, the frequency of mobility and the need of assistance for mobility. Executive function (EF) is one of the most important higher-order cognitive abilities that control and guide goal-directed actions. The aim of the study was to examine the association between $\mathrm{EF}$ and life-space mobility.

A cross-sectional study of 174 community-dwelling persons aged 79 to 93 years living in Central Finland, who participated in the Life-Space Mobility and Active Aging study in 2016. EF was measured with the Trail Making Test (TMT) performance and coded into tertiles according to Delta TMT; TMT-B minus TMT-A (good; Delta-TMT $\leq 92$ s, intermediate; 93-161s and poor; $\geq 162 \mathrm{~s}$ ). Life-space mobility was assessed using the Life-Space Assessment (LSA, range 0-120) and dichotomized to restricted; LSA $<60$ and unrestricted; LSA $\geq 60$ life-space mobility. Adjustments were made for sex, age, years of education and diagnosed depression.

Mean age of the participants was 83 years and $57 \%$ were women. $42 \%$ of the participants had restricted life-space mobility. Logistic regression analysis revealed that people with good EF were less likely to have a restricted life-space mobility compared to those with poor EF (OR 0.394, $95 \%$ CI 0.156-0.996) after adjustment for all covariates.

People with good executive function are less likely to have restricted life-space mobility in old age. Promoting executive function could possibly help maintain mobility and active participation in old age.

\section{EFFECTS OF EXERCISE WITH INCREASED DIETARY PROTEIN ON COGNITION AND QUALITY OF LIFE IN OLDER ADULTS}

M.B. Formica ${ }^{1}$, J. Gianoudis ${ }^{1}$, S. O'Connell ${ }^{1}$, C. Milte ${ }^{1}$, C. Nowson ${ }^{1}$, K. Ellis ${ }^{2}$, R.M. Daly ${ }^{1}$, 1. Deakin University, Melbourne, Victoria, Australia, 2. University of Melbourne, Melbourne, Victoria, Australia

Progressive resistance training (PRT) and dietary protein are essential to optimize muscle health in the elderly, but both factors may also influence brain health/cognitive function via several pathways, including the regulation of various neurotrophic factors [brain-derived neurotrophic factor (BDNF)] and/or the modulation of systemic inflammation. The aim of this study was to investigate the effects of a multi-modal exercise program (aerobic+PRT) combined with a proteinenriched diet achieved through the consumption of lean red meat on cognitive function and health-related quality of life (HR-QoL). Community-dwelling men and women $(n=154)$ aged $65+$ years were randomised to a 24-week intervention consisting of PRT+lean red meat $[2 \times 110 \mathrm{~g}$ raw weight to be consumed on each of the three training days (RT+Meat)] or $\mathrm{PRT}+$ pasta/rice $(\mathrm{RT}+\mathrm{CHO})$. Exercise compliance was no different between groups $(\sim 79 \%)$. Compliance with the meat and $\mathrm{CHO}$ was $87 \%$ and $91 \%$, respectively. After 24 -weeks both groups experienced a similar increase in global cognitive function (z-score change: $\mathrm{RT}+\mathrm{CHO}+0.24 \mathrm{SD}$; RT+Meat $+0.17 \mathrm{SD}$, both $\mathrm{P}<0.001)$. Psychomotor/attention composite scores decreased in the $\mathrm{RT}+\mathrm{CHO}$ after 12 -weeks (interaction, $\mathrm{P}<0.001$ ), but there was no between group difference after 24-weeks. Working memory/learning composite scores increased in the $\mathrm{RT}+\mathrm{CHO}$ compared to $\mathrm{RT}+$ Meat group after 12- and 24-weeks (net difference in z-scores, 0.24-0.27 $\mathrm{SD}$, both $\mathrm{P}<0.001$ ). Executive function, HR-QoL and serum levels of BDNF and inflammation (IL-6, -8, -10, TNF $\alpha$ ) did not change in either group. In conclusion, these findings indicate that the provision of additional protein did not enhance 